\title{
The role of cardiovascular autonomic failure in the differential diagnosis of $a$-synucleinopathies
}

\author{
Fabian Leys $^{1} \cdot$ Gregor K. Wenning $^{1} \cdot$ Alessandra Fanciulli ${ }^{1}$ (i)
}

Received: 13 July 2021 / Accepted: 22 October 2021 / Published online: 24 November 2021

(c) The Author(s) 2021

\begin{abstract}
The $\alpha$-synucleinopathies comprise a group of adult-onset neurodegenerative disorders including Parkinson's disease (PD), multiple system atrophy (MSA), dementia with Lewy bodies (DLB,) and — as a restricted non-motor form — pure autonomic failure (PAF). Neuropathologically, the $\alpha$-synucleinopathies are characterized by aggregates of misfolded $\alpha$-synuclein in the central and peripheral nervous system. Cardiovascular autonomic failure is a common non-motor symptom in people with PD, a key diagnostic criterion in MSA, a supportive feature for the diagnosis of DLB and disease-defining in PAF. The site of autonomic nervous system lesion differs between the $\alpha$-synucleinopathies, with a predominantly central lesion pattern in MSA versus a peripheral one in PD, DLB, and PAF. In clinical practice, overlapping autonomic features often challenge the differential diagnosis among the $\alpha$-synucleinopathies, but also distinguish them from related disorders, such as the tauopathies or other neurodegenerative ataxias. In this review, we discuss the differential diagnostic yield of cardiovascular autonomic failure in individuals presenting with isolated autonomic failure, parkinsonism, cognitive impairment, or cerebellar ataxia.
\end{abstract}

Keywords Autonomic nervous system $\cdot$ Orthostatic hypotension $\cdot$ Cardiovascular autonomic failure $\cdot$ Differential diagnosis $\cdot$ Synucleinopathies

\section{Introduction}

The $\alpha$-synucleinopathies are a group of adult-onset, progressive neurodegenerative disorders characterized by intracellular aggregation of misfolded $\alpha$-synuclein in the peripheral and central nervous system (CNS), including Parkinson's disease (PD), multiple system atrophy (MSA), and dementia with Lewy bodies (DLB) [1, 2]. Oligodendroglial cytoplasmic $\alpha$-synuclein inclusions are characteristic of MSA, which is hence considered an oligodendroglial $\alpha$-synucleinopathy [3]. Depending on the predominance of either cerebellar signs or parkinsonism, a cerebellar (MSA-C) and a Parkinsonian variant (MSA-P) are distinguished [4]. Neuronal $\alpha$-synuclein inclusions, the so-called Lewy bodies and neurites, represent the pathological hallmark of PD and DLB, which are therefore referred to as the Lewy body disorders [5]. Lewy body pathology with deposition of $\alpha$-synuclein

Alessandra Fanciulli

alessandra.fanciulli@i-med.ac.at

1 Division of Neurobiology, Department of Neurology, Medical University of Innsbruck, Anichstrasse 35, Innsbruck 6020, Austria in sympathetic ganglia and peripheral autonomic fibers was also found in individuals with pure autonomic failure (PAF) [6, 7], tying it to the group of $\alpha$-synucleinopathies as a restricted non-motor form [2].

Orthostatic hypotension $(\mathrm{OH})$ is defined by consensus as a sustained fall in systolic/diastolic blood pressure (BP) of $\geq 20 / 10 \mathrm{mmHg}$ within three minutes upon postural change [8]. Orthostatic BP falls causing recurrent spells of dizziness, light-headedness, pre-syncope, or syncope episodes may occur for various reasons [9]. In disorders such as the $\alpha$-synucleinopathies, however, $\mathrm{OH}$ is mostly due to neurodegenerative changes affecting the cardiovascular autonomic nervous system (ANS), i.e. it is of neurogenic origin [10]. The site of ANS lesion is mainly central, or pre-ganglionic, in MSA [3] and peripheral, or post-ganglionic, in PD and DLB (Fig. 1) [11, 12].

Cardiovascular autonomic function tests (CAFT) represent the gold standard for diagnosing neurogenic $\mathrm{OH}$, which is characterized by an insufficient compensatory heart rate (HR) increase despite severe orthostatic BP falls and missing $\mathrm{BP}$ counter regulation under baroreflex stimulation, like during the Valsalva manoeuver [13, 14]. Neurogenic $\mathrm{OH}$ affects every third person with PD $[15,16]$, it is a key diagnostic 
Fig. 1 The cardiovascular autonomic system with diseasespecific sites of lesion. Afferent, central, and efferent components of the cardiovascular autonomic network with possible sites of lesion causing cardiovascular autonomic failure. Deposition of hyperphosphylated $\tau$-protein and $\beta$-amyloid plaques in central autonomic relays, such as the ACC, AMG, and insula presumably cause cardiovascular autonomic impairment in $\mathrm{AD}$ and FTD; predominantly central/pre-ganglionic deposition of oligodendroglial cytoplasmic $\alpha$-synuclein inclusions occurs in MSA, while PD and DLB are characterized by predominantly peripheral/ post-ganglionic deposition of neuronal $\alpha$-synuclein inclusions. Central structures, particularly the dorsal motor nucleus of vagus, are also affected in $\mathrm{PD} /$ DLB, whereas in MSA the neurodegenerative process may propagate from the pre- to the post-ganglionic branch during the disease course. $A C C$ anterior cingulate cortex; $A D$ Alzheimer's disease; $A M G$ amygdala; $D L B$ dementia with Lewy bodies; FTD frontotemporal dementia; HPT hypothalamus; $I X$ glossopharyngeal nerve; MSA multiple system atrophy; $P D$ Parkinson's disease; $X$ vagal nerve. Created with Microsoft Office PowerPoint 2016, in part using adapted Servier Medical Art images. (https://smart.servi er.com/)

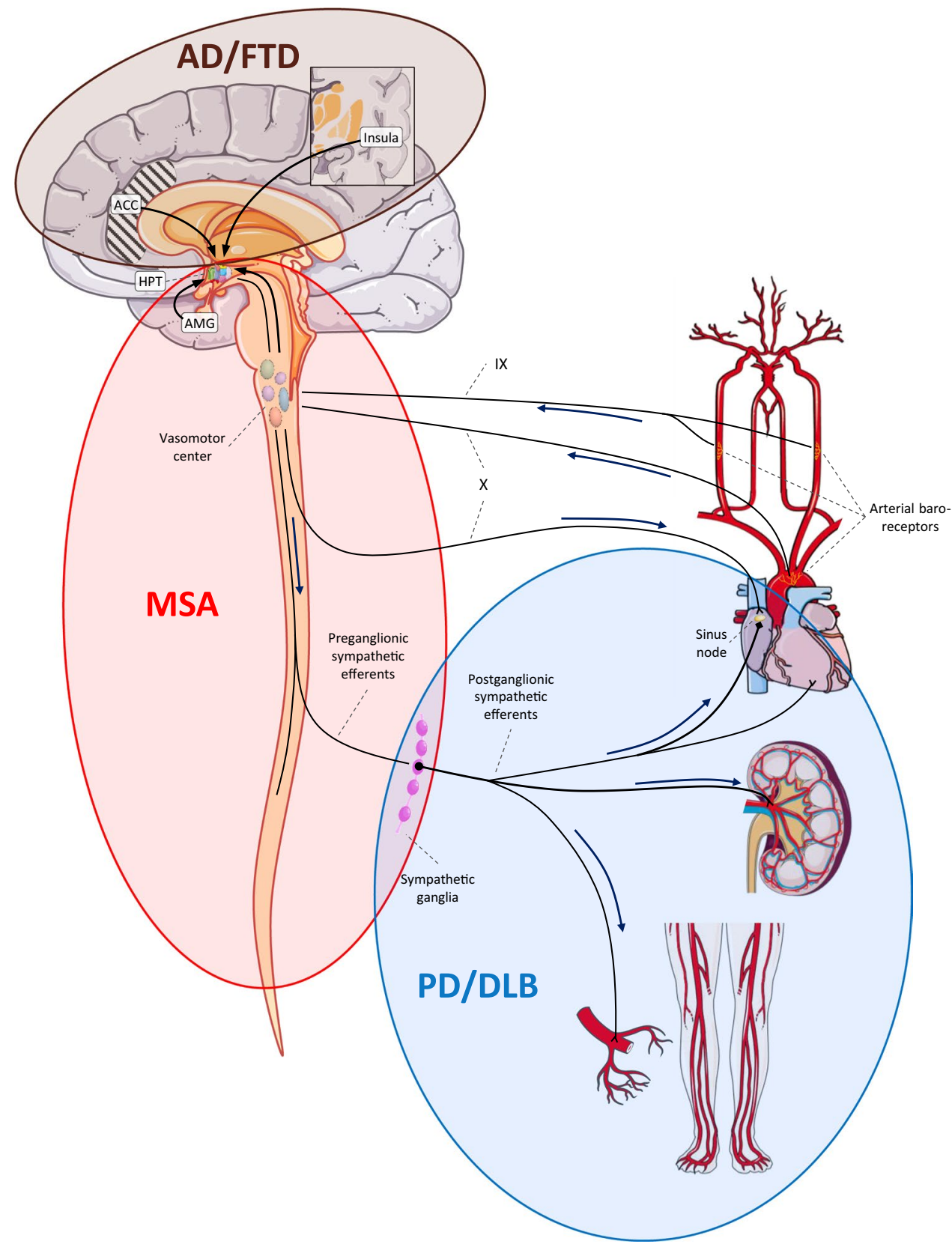

criterion for MSA $[4,17,18]$, supports the diagnosis of DLB [19-21], and defines the disease in PAF [22]. High BP levels while supine and asleep, i.e. supine and nocturnal hypertension [23], post-prandial hypotension [24] and exerciseinduced hypotension [25] may accompany neurogenic $\mathrm{OH}$ in about $50 \%$ of individuals, with major prognostic and therapeutic implications [10].

Distinguishing one $\alpha$-synucleinopathy from the other, but also separating them from related disorders, such as the tauopathies or other neurodegenerative ataxias, is often challenging, especially at disease onset due to overlapping presentation [26, 27]. Misdiagnosis may result in inadequate treatment, erroneous or missed inclusion in clinical trials. Given the narrow window of opportunity in which candidate neuroprotective therapies might help modifying the relentless disease progression of the $\alpha$-synucleinopathies, a timely and accurate diagnosis is of utmost importance for an appropriate counselling and improved health care.

This review focuses on the role of cardiovascular autonomic failure in the differential diagnostic work-up of individuals presenting with neurological features that potentially indicate an incipient $\alpha$-synucleinopathy, i.e. isolated autonomic failure, parkinsonism, cognitive impairment, or cerebellar ataxia. 


\section{Methods}

References were identified by searching the PubMed database up to April 2021, combining keywords for disease entities, disorders, or non-ANS-related clinical features with ANS-related and other keywords as follows: ["Alzheimer dementia" OR "Alzheimer disease" OR "ataxia" OR "CANVAS" OR "cerebellar ataxia" OR "cognitive impairment" OR "corticobasal degeneration" OR "corticobasal syndrome" OR "dementia with Lewy bodies" OR "dementia" OR "fragile X tremor ataxia syndrome" OR "Friedreich ataxia" OR "frontotemporal dementia" OR "Lewy body disorders" OR "multiple system atrophy" OR "neurodegenerative dementia" OR "parkinsonism" OR "Parkinson's disease" OR "progressive supranuclear palsy" OR "pure autonomic failure" OR "spinocerebellar ataxia" OR "sporadic adult onset ataxia" OR "sporadic late onset ataxia" OR "synucleinopathies" OR "tauopathies"] AND ["autonomic" OR "autonomic dysfunction" OR "autonomic failure" OR "autonomic function" OR "cardiovascular autonomic dysfunction" OR "cardiovascular autonomic failure" OR "differential diagnosis" OR "exercise induced hypotension" OR "heart rate variability" OR "isolated autonomic failure" OR "natural history" OR "neurogenic orthostatic hypotension" OR "nocturnal hypertension" OR "orthostatic hypotension" OR "postprandial hypotension" OR "supine hypertension" OR "syncope"].

Meta-analyses, systematic and narrative reviews, crosssectional, prospective and retrospectives original studies, as well as case reports, were included. Additional data were collected by hand-searching references from selected articles. We also included historically noteworthy and conceptually related articles. We only considered articles published in English language.

\section{Isolated autonomic failure}

PAF was defined by consensus in 1996 as a sporadic disorder characterized by idiopathic neurogenic $\mathrm{OH}$ with evidence of more widespread autonomic failure, but no other neurological features [22]. At this time, it was already known that low supine plasma norepinephrine (NE) levels, indicating a widespread post-ganglionic sympathetic denervation, are characteristic of PAF [6, 7], as well as that some individuals will eventually develop a manifest CNS involvement during the disease course [22]. For this reason, a history of at least five years of isolated autonomic failure without development of other neurological features was proposed as additional criterion to increase the diagnostic accuracy for PAF [28].

In recent years, one prospective North American multicenter study as well as three retrospective single-center studies from two independent research consortia investigated the natural history of PAF and reported a phenoconversion to another $\alpha$-synucleinopathy in approximately one-third of individuals [2, 29-31]. The phenoconversion rate to MSA was $7-28 \%$ and to Lewy body disorders $4-26 \%$, depending on the duration of the clinical follow-up (see Table 1) [2, 29-31].

Individuals who maintained the PAF phenotype ("stable PAF") or rather phenoconverted to another $\alpha$-synucleinopathy with overt CNS involvement ("CNS

Table 1 Natural history studies of PAF

\begin{tabular}{|c|c|c|c|c|c|}
\hline & Kaufmann et al. [2] & Singer et al. [29] & & Coon et al. [30] & Giannini et al. [31] \\
\hline Design/cohort & $\begin{array}{l}\text { Prospective multi-center (NYUMC, } \\
\text { NIH, VU, Mayo Clinic, Beth Israel; } \\
\text { US) }\end{array}$ & \multicolumn{3}{|c|}{ Retrospective single-center (Mayo Clinic, US) } & $\begin{array}{l}\text { Retrospective single- } \\
\text { center (Bologna, } \\
\text { Italy) }\end{array}$ \\
\hline Published & 2017 & 2017 & & 2020 & 2018 \\
\hline Follow-up, years & 4 & any & $\geq 3$ & $\geq 5$ & $\geq 5$ \\
\hline Sample size, $\mathrm{n}$ & 74 & 318 & 78 & 275 & 50 \\
\hline Conversion rate, $\mathrm{n}(\%)$ & $25(34)$ & $37(12)^{*}$ & $37(47)^{*}$ & $67(24)$ & $16(32)^{* *}$ \\
\hline $\begin{array}{l}\text { Conversion to Lewy body } \\
\text { disorders, } \mathrm{n}(\%)\end{array}$ & $19(26)^{+}$ & $11(4)$ & $11(14)$ & $33(12)$ & $3(6)^{++}$ \\
\hline Conversion to MSA, n (\%) & $6(8)$ & $22(7)$ & $22(28)$ & $34(12)$ & $10(20)$ \\
\hline
\end{tabular}

$D L B$, dementia with Lewy bodies; $M S A$, multiple system atrophy; $N I H$, National Institute of Health Bethesda; NYUMC, New York University Medical Center; $P D$, Parkinson's disease; $U S$, United States; $V U$, Vanderbilt University

*Including $n=4$ individuals with development of clear motor involvement, but indeterminate diagnosis

** Including $n=3$ individuals with undefined parkinsonism

${ }^{+}$Including $n=13$ (18) DLB and $n=6$ (8) PD phenoconverters

${ }^{++}$Including $n=2$ (4) DLB and $n=1$ (2) PD phenoconverters

Classification of phenoconversion to PD or DLB not specified in [29, 30] 
$\alpha$-synucleinopathy") were found to exhibit distinct baseline features, which ultimately foreshadowed the disease course [2, 29-31]. The current understanding is that features suggestive of CNS involvement, such as dream enacting behavior and subtle motor signs, as well as earlier or more severe manifestation of additional autonomic disturbances, indicate a future phenoconversion to a CNS $\alpha$-synucleinopathy [2, 29-31]. Together with indices referring to either a predominant central or peripheral pattern of ANS lesion, it may be possible to predict whether an individual will eventually phenoconvert to MSA or a Lewy body disorder already at prodromal disease stages (see Table 2) [2, 29-31].

In abovementioned studies, both individuals with stable PAF and future phenoconverters to Lewy body disorders had a later disease onset and showed low baseline supine plasma NE levels, as well as an insufficient orthostatic HR increase, indicating a peripheral pattern of ANS lesion [2, 29-31]. However, Lewy body phenoconverters differed from individuals with stable PAF for a preserved orthostatic NE rise (indicating partially preserved post-ganglionic sympathetic fibers), presence of subtle motor parkinsonian signs, hyposmia, as well as constipation and an earlier occurrence of urinary dysfunction (see Table 2) [2, 29-31]. On the other hand, earlier onset of neurogenic $\mathrm{OH}$, preserved orthostatic HR increase, higher supine and orthostatic NE levels, a preganglionic sweat loss pattern (all consistent with a central ANS lesion pattern), as well as the presence of subtle parkinsonian or cerebellar signs, stridor, more severe urinary dysfunction and constipation, but preserved olfaction, predicted future phenoconversion to MSA [2, 29-31].

Supportive imaging data are scarce, with results from cardiac ${ }^{123}$ I-MIBG-SPECT only available in one study [31]. Reduced ${ }^{123}$ I-MIBG-uptake, indicating sympathetic cardiac denervation, was observed only in individuals with stable PAF or future Lewy body phenoconverters, while MSA phenoconverters showed normal cardiac ${ }^{123}$ I-MIBG-uptake, indicating a pre-ganglionic site of ANS lesion [31]. This was also the case in three individuals presenting with isolated neurogenic $\mathrm{OH}$, who had normal cardiac ${ }^{123}$ I-MIBG findings and later phenoconverted to MSA, which was neuropathologically confirmed in two [32]. The utility of DAT-SPECT and conventional MRI as predictors of phenoconversion is yet unknown [34]. Presynaptic nigrostriatal denervation as indicated by pathological DAT-SPECT findings and loss of dorsolateral nigral hyperintensity at MRI may be predictors for both MSA and Lewy body phenoconversion [35],

Table 2 Features associated with phenoconversion from PAF to MSA versus Lewy body disorders, or both

\begin{tabular}{|c|c|c|}
\hline & Multiple system atrophy & Lewy body disorders \\
\hline Hemodynamic indices & $\begin{array}{l}\text { Earlier OH onset (mainly } 50 \mathrm{~s} \text { ) }[2,30] \\
\text { Preserved HR increase to tilt, i.e.: } \\
\text { - after } 1 \mathrm{~min}[30] \text {; } \\
\text { - > } 10 \mathrm{bpm} \text { after } 3 \min [2]\end{array}$ & Later $\mathrm{OH}$ onset (mid to end $60 \mathrm{~s}$ ) $[2,29,30]$ \\
\hline Imaging and wet biomarkers & $\begin{array}{l}\text { Supine NE levels }>100 \mathrm{pg} / \mathrm{ml}[29,30] \text { or }>110 \mathrm{pg} / \mathrm{ml}[2] \\
\text { Higher orthostatic NE levels [29] } \\
\text { Normal cardiac }{ }^{123} \text { I-MIBG uptake* }[31,32] \\
\text { Lower maximum-ThT-fluorescence at PMCA assay and higher Nfl } \\
\text { levels in CSF [33] } \\
\text { Putaminal/infratentorial abnormalities and increased putaminal/ } \\
\text { MCP diffusivity at MRI* [34] } \\
\text { Orthostatic NE rise }>65 \mathrm{pg} / \mathrm{ml}[29,30] \\
\text { Presynaptic nigrostriatal denervation at DAT-SPECT }{ }^{*}[34,35] \\
\text { Loss of dorsolateral nigral hyperintensity at MRI* }[35]\end{array}$ & \\
\hline $\begin{array}{l}\text { Other autonomic or CNS- } \\
\text { related signs and features }\end{array}$ & $\begin{array}{l}\text { Preserved olfactory function }{ }^{* *}[2] \\
\text { Urinary dysfunction }[2,29,30] \text { and constipation }[2] \text { of moderate to } \\
\text { severe degree } \\
\text { Preganglionic sweat loss pattern }[29,30] \\
\text { Stridor [31] } \\
\text { Constipation }[2,30] \text { (contrasting evidence with [31]) } \\
\text { Earlier onset of urinary dysfunction and intermittent catheterization } \\
\text { Dream enactment behavior, i.e. probable RBD }[2,30] \text { and earlier ons } \\
\text { Subtle motor signs }[2,29,30] \text {, frequent falls }{ }^{* * *}[31]\end{array}$ & $\begin{array}{l}\text { [31] } \\
\text { et of VPSG-confirmed RBD }\end{array}$ \\
\hline
\end{tabular}

$C N S$, central nervous system; $H R$ heart rate; $M C P$, middle cerebellar peduncle; $M S A$, multiple system atrophy; $N E$, norepinephrine; $N f$, neurofilament light chain; $O H$, orthostatic hypotension; $P A F$, pure autonomic failure; $P M C A$, protein misfolding cyclic amplification; $R B D$, REM sleep behavior disorder; $T h T$, thioflavin T; $V P S G$, video-polysomnography

*Not systematically studied

** In combination with probable RBD

**** Individuals later phenoconverting to MSA or Lewy body disorders analyzed together in [31] 
whereas putaminal and infratentorial abnormalities, as well as increased putaminal and middle cerebellar peduncle diffusivity on MRI, may prove to be predictors of MSA phenoconversion only [34].

A very recent study prospectively followed a well-characterized cohort of PAF individuals to determine the role of $\alpha$-synuclein oligomers and neurofilament light chain ( $\mathrm{Nfl}$ ) in cerebrospinal fluid as markers of phenoconversion [33]. As previously shown for the distinction of MSA from Lewy body disorders [36], higher Nfl measurements and lower maximum-thioflavin-fluorescence in the protein misfolding cyclic amplification (PMCA) assay of baseline cerebrospinal fluid accurately distinguished future phenoconversion to MSA versus Lewy body disorders or stable PAF [33].

The role of other features of cardiovascular autonomic failure, such as supine hypertension, post-prandial- or exercise-induced hypotension, in predicting future phenoconversion to a central $\alpha$-synucleinopathy in people presenting with isolated autonomic failure has not been extensively studied yet, but it is probably limited.

\section{Parkinsonism}

Parkinsonism is characterized by the presence of bradykinesia and at least one additional extrapyramidal feature out of rigidity, rest tremor or postural instability [16]. Beyond PD, which accounts for the majority of cases [37], atypical parkinsonian disorders including DLB, MSA, progressive supranuclear palsy (PSP), and corticobasal syndrome (CBS) have to be considered in the diagnostic work-up of individuals presenting with parkinsonism [38].

Both PSP and CBS belong to the tauopathies, a group of heterogeneous neurodegenerative disorders characterized by abnormal intracerebral aggregation of $\tau$-protein in neurons and glia $[39,40]$. While features of otherwise unexplained autonomic dysfunction qualify as exclusion criteria for the diagnosis of CBS [40], autonomic disturbances in PSP mainly involve the urogenital domain and are generally less common and severe compared to the $\alpha$-synucleinopathies $[34,39,41,42]$. Although incipient signs of cardiovascular dysfunction, including loss of the physiological nocturnal BP fall and post-prandial hypotension, have been reported in PSP, severe orthostatic BP falls of $\geq 30 / 15 \mathrm{mmHg}$ do not usually occur, especially at early stages [42-46]. More recent reviews of large neuropathologically-proven PSP series found $\mathrm{OH}$ to occur only in single cases (in which other frequent causes of both neurogenic and non-neurogenic $\mathrm{OH}$ were not excluded systematically), confirming that the cardiovascular sympathetic system is largely spared in PSP [41, 47]. Beyond other clinical and instrumental features, the presence of overt cardiovascular autonomic failure therefore yields a high negative predictive value for a diagnosis of both PSP and CBS and helps distinguishing parkinsonian syndromes due to an underlying tauopathy from those due to $\alpha$-synucleinopathies [34, 39-41].

On the other hand, the presence of autonomic failure may often make it difficult to differentiate one $\alpha$-synucleinopathy from the other, especially at early stages, where both people with Lewy body disorders and MSA may show good L-Dopa responsiveness [16, 27, 48]. Recent neuropathological series of individuals diagnosed in-life with MSA following the Gilman 2008 criteria [4] reported a diagnostic accuracy of 62 to $79 \%$, with Lewy body disorders accounting for the most common misdiagnosis $[41,49]$. A review of medical records identified severe, widespread autonomic failure as the main reason for Lewy body disorder individuals to receive an ante-mortem diagnosis of MSA [49]. Due to the proposed different site in ANS lesion [3, 11], hemodynamic indices of CAFT were often studied for their diagnostic yield in discriminating MSA from Lewy body disorders, particularly PD [20]. Significant differences were frequently reported, but inconsistencies and substantial overlap hinder a reliable differentiation between PD and MSA based on CAFT indices alone $[14,27,34,50,51]$. Cardiovascular autonomic failure is generally more frequent and severe in MSA than in Lewy body disorders [15, 17, 18, 20, 46]. However, the presence of neurogenic $\mathrm{OH}$ remains by itself of limited diagnostic value in single cases, while its early development, in conjunction with additional urological autonomic features and rapid progression of motor impairment yields a higher accuracy for separating MSA from PD [52, 53]. By contrast, at more advanced disease stages, every third patient with PD may develop neurogenic $\mathrm{OH}$ and neither its presence, nor its severity remain specific of MSA [15, 52, 53].

Consistent with the predominantly peripheral ANS involvement in the Lewy body disorders [11], cardiac sympathetic denervation at ${ }^{123} \mathrm{I}$-MIBG-SPECT or ${ }^{18} \mathrm{~F}$-Dopamine-PET has been shown in PD and DLB individuals with and without neurogenic $\mathrm{OH}$ [54-57]. In absence of cardiomyopathy or other causes of peripheral autonomic neuropathy, the presence of cardiac sympathetic denervation is therefore considered a supportive imaging criterion for both a PD and DLB diagnosis [19, 55-59]. By contrast, most individuals with MSA have an intact cardiac innervation [55-57]. Due to a possible prion-like propagation of $\alpha$-synuclein pathology from the pre- to the post-ganglionic sympathetic branch [26], however, cardiac sympathetic denervation may be found in people with MSA as well, especially at advanced stages, limiting the implementation of cardiac sympathetic imaging in its diagnostic work-up [4, $55,58]$.

Measurement of plasma NE levels may also be helpful to differentiate between MSA and Lewy body disorders [14]. Supine plasma NE levels in MSA are comparable to those of people with non-neurogenic $\mathrm{OH}$, whereas significantly lower levels were demonstrated in individuals with neurogenic $\mathrm{OH}$ 
due to Lewy body disorders [14]. To date, however, clear cut-off values with optimized sensitivity and specificity are still missing.

Supine and nocturnal hypertension are more common and severe in individuals with neurogenic $\mathrm{OH}$ due to MSA than PD [23, 50, 60], and presumably DLB, but same as for post-prandial- and exercise-induced hypotension, yield a limited value for differentiating one $\alpha$-synucleinopathy from the other.

\section{Cognitive impairment}

Cognitive impairment is defined by any deterioration in cognitive performance from the individual previous level that is significant enough to interfere with the independent daily functioning [61]. Alzheimer's disease (AD) represents the most common cause of cognitive impairment [61]. Frontotemporal dementia (FTD) and Lewy body disorders (i.e. DLB and PD dementia) respectively account for the second leading cause of neurodegenerative cognitive decline under and above the age of 65 [61, 62].

The FTD spectrum includes various clinical subtypes [62], including its behavioral variant (bvFTD). Signs of both sympathetic and cardio-vagal dysfunction have been reported in individuals with the bvFTD and AD [63-66], possibly due to affection of telencephalic structures involved in the central autonomic network (mainly the anterior cingulate- and insular cortex; see Fig. 1) [67, 68]. Compared to healthy elderly controls, a higher prevalence of post-prandial hypotension, but not of $\mathrm{OH}$, was also shown in AD [69]. Supine BP readings of individuals with AD provided contrasting results as to the presence of supine hypertension, which was not found in individuals with bvFTD $[64,66]$. To the best of our knowledge, there are no systematic studies on cardiovascular autonomic function in people with other FTD variants, such as agrammatic- or semantic primary progressive aphasia and motor-neuron disease-related FTD.

Overall, current data on cardiovascular autonomic failure in tauopathy-related cognitive impairment are scarce, provide contrasting results, and remain limited by a substantial heterogeneity [68]. The frequency of overt neurogenic $\mathrm{OH}$ in people with clinically ascertained tauopathies remained low in the reviewed studies and the role of age-related confounders, such as cardiovascular comorbidities, medications, mixed pathology, or aging itself, remains to be clarified [67, $68,70]$.

While the exact neuropathological substrate of cardiovascular autonomic dysfunction in the tauopathies has not yet been unravelled, both serological and imaging studies indicate intact post-ganglionic sympathetic fibers in these diseases [55-57, 64]. Normal supine and standing plasma $\mathrm{NE}$ levels were reported in individuals with $\mathrm{AD}$, as well as in all but one with bvFTD [64], while ${ }^{123}$ I-MIBG-SPECT studies showed intact cardiac sympathetic innervation in people with AD [55-57]. Cardiac ${ }^{123}$ I-MIBG-SPECT has been therefore proposed as useful tool to differentiate both bvFTD and AD from Lewy body disorders related dementia [55-57]. Ultimately, the presence of otherwise unexplained neurogenic $\mathrm{OH}$ with evidence of post-ganglionic sympathetic denervation at ${ }^{123}$ I-MIBG-SPECT in a cognitively declined individual points towards an $\alpha$-synucleinopathy, most likely DLB [55, 67, 68, 71].

Although the current consensus criteria consider dementia as a non-supportive feature pointing against a diagnosis of MSA [4], there is growing evidence that cognitive impairment in fact represents an important non-motor feature affecting about one-third of people with MSA [27, 41, 72]. Recent neuropathological series found that up to one in seven individuals who received an ante-mortem MSA diagnosis in fact turned out to suffer from DLB at post-mortem examination $[41,49]$. Early and severe autonomic failure and an "atypical" presentation with only mild cognitive impairment were found to represent the main factors determining an ante-mortem misdiagnosis of MSA in DLB individuals [49]. Beyond DLB-specific clinical red flags, such as early, rapidly progressive cognitive decline with marked fluctuations in attention and alertness, ancillary findings indicating a peripheral pattern of ANS lesion, i.e. cardiac denervation at ${ }^{123}$ I-MIBG-SPECT, may help the differential diagnosis of DLB from MSA, even in cases with a rather "atypical" presentation [14, 19, 34, 41, 49, 56, 57].

By contrast, PD dementia and DLB present largely overlapping features of cardiovascular autonomic failure, despite this is considered more prevalent and severe in the latter [20, 55]. Ultimately, the arbitrary cut-off of one year ("1-year rule") remains useful in discriminating DLB from PD with dementia in clinical practice [19].

\section{Cerebellar ataxia}

Ataxia, originally from ancient Greek meaning "absence of order", is defined by an impaired coordination of voluntary movements, which may result from sensory, vestibular or cerebellar impairment, or a combination thereof [73]. Individuals may present with acquired ataxia due to a wide range of symptomatic causes, which should be considered in the diagnostic work-up and sought for with a careful history taking, neurological examination, imaging, and laboratory tests [74]. In absence of apparent exogenous or endogenous nongenetic causes, ataxia may be hereditary or idiopathic [74].

The group of idiopathic late-onset cerebellar ataxias (ILOCA) includes MSA-C as well as sporadic adult-onset ataxia of unknown aetiology (SAOA) [74]. According to the current definition, SAOA is a clinical diagnosis of exclusion, which is given if acquired and genetic causes (either by exclusion of a causative genetic mutation or a presumably 
negative family history, if genetic testing is not available) are ruled out and the diagnostic criteria for MSA [4] are not met [74]. Absence of severe autonomic failure over the disease course eventually represents the discriminating element between SAOA and MSA [74, 75]. Studies however showed that a substantial proportion of people initially labelled as SAOA may develop overt autonomic failure during the disease course, i.e. phenoconvert to MSA [75-77]. In contrast to previous reports that milder urogenital symptoms, i.e. urinary urgency, frequency, or sexual dysfunction, already discriminate MSA from SAOA at initial presentation [75], a high prevalence of these was recently found at baseline examination of individuals with SAOA who did not convert to MSA during follow-up [76]. As early as 2007, it was shown that mild cardiac parasympathetic abnormalities may be observed in up to $58 \%$ of SAOA individuals at CAFTs [78], but no relevant orthostatic BP falls fulfilling the criteria for neurogenic $\mathrm{OH}$ [8]. Milder falls in BP upon standing, as well as pathological cardio-vagal responses to the Valsalva manoeuver and deep breathing, may in fact indicate future phenoconversion to MSA, whereas normal CAFT results at initial assessment may direct to genetic testing, especially if a SAOA diagnosis was based on a negative family history $[74,76]$. Cardiovascular autonomic failure can therefore be considered the most specific sign guiding the differential diagnosis of ILOCAs towards MSA [76]. However, because its sensitivity increases with the disease duration, the absence of overt cardiovascular autonomic failure at early stages does not exclude a phenoconversion to MSA later on [74-76].

Both male and, less frequently, female individuals with 55-200 CGG repeat expansions of the FMR1 gene may develop the fragile $\mathrm{X}$-associated tremor ataxia syndrome (FXTAS), which clinically manifests with progressive cerebellar ataxia, prominent tremor, as well as cognitive impairment, neuropathy, L-Dopa-unresponsive parkinsonism and autonomic failure [74, 79-81]. The latter may include urogenital and gastrointestinal dysfunction, as well as cardiovascular autonomic features, e.g. post-prandial hypotension and symptomatic neurogenic OH [79-81]. Due to the overlap in symptoms, the European MSA study group screened for FMR1 repeat expansions in a large cohort of clinically diagnosed MSA individuals and found them in $\leq 1 \%$ of cases ( $n=4 / 426$ ), but in $4 \%$ of those presenting with a MSA-C phenotype $(n=3 / 76)$ [80]. While urogenital dysfunction was found in all misdiagnosed cases, neurogenic $\mathrm{OH}$ was present only in one [80]. To date, evidence of cardiovascular autonomic failure in FXTAS is limited to few case reports [79-81] and despite occurring less frequently than urogenital dysfunction, it may still lead to a MSA misdiagnosis, especially if cerebellar features predominate [80]. An unusually slow progression, predominant intention tremor or peripheral neuropathy, as well as a familial clustering of mental retardation, may eventually prompt genetic testing for FMR1 repeat expansions in people with ILOCA [80].

GAA triplet expansions in the FXN gene cause the single most common hereditary cause of ataxia in Caucasian natives, that is Friedreich's ataxia (FRDA) [82]. Recent evidence from a single-center, but well-characterized cohort of genetically confirmed FRDA individuals and controls without diabetes mellitus or symptomatic cardiomyopathy, showed a high prevalence of urinary symptoms [82], but unremarkable CAFT results, indicating that cardiovascular autonomic failure is not typical of the FRDA clinical spectrum $[82,83]$. However, because diabetes mellitus and hypertrophic cardiomyopathy may frequently occur in people with FRDA, diabetic autonomic neuropathy or nonneurogenic forms of $\mathrm{OH}$ may develop in some individuals, especially in case of poor glycaemic control, overt heart failure or anti-hypertensive treatments [82].

The cerebellar ataxia, neuropathy, vestibular areflexia syndrome (CANVAS) recently emerged as an additional cause of late-onset ataxia [84]. Cortese et al. identified biallelic intronic AAGGG expansions in the RFC1 gene as CANVAS' underlying cause [85]. The phenotypical characterization of a large cohort of people with genetically confirmed CANVAS showed that autonomic dysfunction can be part of the CANVAS clinical spectrum, with up to $13 \%$ of individuals reporting symptoms suggestive of $\mathrm{OH}[85,86]$. At autonomic function testing, every fourth individual of the abovementioned CANVAS cohort had a pathological regulation of cardio-vagal reflexes and every third showed sympathetic abnormalities, either during skin sympathetic reflex, handgrip testing or upon orthostatic challenge, but neither the hemodynamic indices, applied reference values nor the prevalence of $\mathrm{OH}$ were reported [86]. In contrast to MSA, however, autonomic dysfunction in CANVAS individuals was generally considered mild, developed late in the disease course, and rarely resulted in significant disability, i.e. overt neurogenic $\mathrm{OH}[84,86]$. Biallelic AAGGG expansions were also not identified in clinically diagnosed MSA individuals [4], suggesting that the slower disease progression and additional signs, such as prominent vestibular dysfunction and sensory neuropathy, prevent people with CANVAS from being misdiagnosed with MSA [84, 86, 87].

The group of autosomal dominant spinocerebellar ataxias (SCA) includes various subtypes to be considered in the differential diagnosis of late-onset cerebellar ataxia, even in the case of negative family history $[74,88]$. Because autonomic dysfunction as well as parkinsonism may occur in some SCA subtypes (e.g. SCA 1, 2, 3, 6, and 17), difficulties may arise in the differential diagnosis of both MSA-C and MSA-P [88]. Only recently, it was shown that $7.3 \%(n=22 / 302)$ of individuals with possible and probable MSA [4] in fact carried mutations for dentatorubropallidoluysian atrophy, SCA $1,2,3,6$, and 17 , with the latter accounting for $60 \%$ of the 
genetic mimicries $(n=13 / 22)$ [89]. Urinary incontinence, or at least frequency, were present in all misdiagnosed cases, whereas almost half $(n=10 / 22 ; 80 \%$ of those with SCA 17 mutations) showed severe BP falls of $\geq 30 / 15 \mathrm{mmHg}$ or reported orthostatic dizziness [89]. Two other studies found that overt $\mathrm{OH}$ did not occur in a SCA 2 only-as well as a mixed cohort including individuals with SCA 1, 2, 3, 6 and FRDA mutations, with however pathological cardio-vagal responses to the Valsalva manoeuver and deep breathing in more than the half of cases [76, 90]. Interestingly, earlier reports indicated that cardiovascular autonomic failure with $\mathrm{OH}$ may be found in up to $25 \%$ people with SCA 3 mutations, with one study also reporting cardiac sympathetic denervation at ${ }^{123}$ I-MIBG-SPECT $[91,92]$. At present, neurogenic $\mathrm{OH}$ seems to yield a limited diagnostic accuracy for distinguishing MSA-C from autosomal dominant cerebellar ataxia, particularly SCA 3 and 17, but further studies in larger independent cohorts with combined CAFT and cardiac ${ }^{123}$ I-MIBG-SPECT are needed to clarify this issue [34, $76,89-92]$. Whereas it is not generally recommended to perform genetic testing in individuals with clinically established MSA [4], this may be considered in case of positive family history, isolated cerebellar atrophy at neuroimaging or mild, non-progressive forms of autonomic failure [88, 89].

Neither supine-/nocturnal hypertension nor post-prandialor exercise-induced hypotension currently contribute to the differential diagnostic work-up of cerebellar ataxia.

\section{Summary}

Even after five years of disease duration, a significant proportion of individuals initially presenting with autonomic failure will eventually phenoconvert to a manifest CNS $\alpha$-synucleinopathy, raising the question whether PAF represents an entity of its own or rather a prodromal stage of MSA, PD, or DLB [2, 29-31]. In this context, "isolated" would be a more cautious term to use instead of "pure" autonomic failure, leaving the possibility open for a future clinical phenoconversion. The predominant site of autonomic lesion, i.e. central versus peripheral, together with other clinical and wet biomarkers was shown to reliably predict phenoconversion from isolated autonomic failure to MSA or a Lewy body disorder, either PD or DLB [2, 29-31]. Early identification and differentiation of these individuals are of high scientific and clinical interest, since it may enable their enrolment into disease-modifying clinical trials at a stage in which the neurodegenerative process is already present, but has not caused any motor disability yet $[29,30]$. Future studies with large sample sizes and longer follow-up time are needed to confirm the existing-, but also integrate additional imaging and wet predictors of phenoconversion. On the other hand, it is intriguing to speculate that individuals retaining a stable PAF phenotype may have genetic traits protecting them from developing CNS $\alpha$-synuclein aggregates, equally deserving further investigations to understand the mechanisms underlying $\alpha$-synuclein-related neurodegenerative processes [2].

In individuals presenting with parkinsonism, the presence of overt cardiovascular autonomic failure allows to exclude both PSP and CBD with high specificity [34, 39-41], while the pattern of ANS lesion distribution in conjunction with the time course of autonomic onset may guide the differential diagnosis between MSA and Lewy body disorders [3, $11,14,34,41,52-57]$.

Compared to parkinsonism, there is only limited data available in cognitively impaired individuals, especially with regard to tauopathy-related dementia [34]. While it remains to be clarified whether $\tau$-related neuropathological changes involving central autonomic relays may cause overt cardiovascular autonomic failure [67, 68, 70], an otherwise unexplained neurogenic $\mathrm{OH}$ with evidence of cardiac sympathetic denervation yields a high negative predictive value for tauopathy-related dementia and rather points to an $\alpha$-synucleinopathy-related dementia [55, 67, 68, 71]. PD with dementia and DLB are still separated by the one-year rule [19].

In individuals with ILOCA, presence of cardiovascular autonomic failure with $\mathrm{OH}$ allows to distinguish MSA-C from SAOA [34, 74]. By contrast, with exception of FRDA and SCA 2, cardiovascular autonomic failure may have a limited contribution to the differential diagnosis of MSA versus hereditary cerebellar ataxias, particularly SCA 3 and 17 [34, 76, 80, 84, 89]. Mild forms of autonomic failure have been reported in CANVAS and FXTAS, which however represent unlikely MSA misdiagnoses, because of additional disease-specific characteristics and a generally slower disease progression $[80,84,86]$. An atypical MSA presentation, with mild or non-progressive forms of autonomic failure, may eventually raise the suspicion of a genetic lookalike and prompt additional testing, even in individuals with negative family history [76, 80, 88, 89].

Despite representing important clinical findings with major therapeutic implications [93], supine and nocturnal hypertension, as well as post-prandial- and exercise-induced hypotension, currently do not aid the differential diagnosis of autonomic disorders. The spectral analysis of HR variability in the time and frequency domain represents another measure of cardiovascular autonomic function [94-96]. Due to considerable overlaps among neurodegenerative disorders, spectral HR analysis does not provide reliable implications for their differential diagnostic work-up either [94, 95, 97]. However, a reduced HR variability in the absence of overt neurogenic $\mathrm{OH}$ may indicate incipient baroreflex dysfunction and eventually make physicians alert of an increased susceptibility to BP active medications in affected individuals. 
The major shortcomings of the reviewed literature were the lack of validation in neuropathologically or genetically confirmed series. Significant heterogeneity in methodology, i.e. discrepancies in the definition of neurogenic $\mathrm{OH}$ or the performance of CAFT, but also the generally scant or very early biomarker data (e.g. plasma NE measurements; ${ }^{123}$ I-MIBG-SPECT) further limit the generalizability of the reported findings, identifying the areas of future research.

In clinical practice, CAFT should be carried out following the international consensus recommendations $[13,97$, 98]. The prevalence of $\mathrm{OH}$, comorbidities, and polypharmacy typically increases with age $[9,10,99,100]$. When screening elderly subjects, attention must be paid to the fact that non-neurogenic factors, such as concomitant heart failure, other cardiac diseases, medication or other frequent causes of neurogenic $\mathrm{OH}$, e.g. diabetic neuropathy, may influence the global cardiovascular performance [9, 10, 99, 100]. Advanced motor- and cognitive impairment may also negatively impact on the execution of CAFTs, especially those examinations requiring an individual's active cooperation, such as the Valsalva manoeuver or deep breathing [13, 65, 76, 91]. This may lead to false abnormal CAFT results. Moreover, cognitive impairment may interfere with the perception and the communication of symptoms of orthostatic intolerance, with a substantial risk of eventually under-recognizing the presence of cardiovascular autonomic failure or underestimating its impact on the quality of life of demented people [64-66].

In conclusion, the presence of cardiovascular autonomic failure significantly contributes to the diagnostic work-up of the $\alpha$-synucleinopathies and related disorders. Given its rapidly progressive, fatal disease course, MSA emerges as the most crucial differential diagnosis to recognize or exclude. Beyond the differential diagnostic implications arising from the presence of cardiovascular autonomic failure, the establishment of an appropriate therapeutic regimen is of utmost importance in order to reduce the symptomatic burden and avoid short and long-term complications of untreated BP dysregulation [93]. Screening for neurogenic $\mathrm{OH}$ at bedside with simple supine to standing HR and BP measurements $[101,102]$ and confirmation of the diagnosis with CAFT should therefore always be considered if cardiovascular autonomic failure is suspected.

\footnotetext{
Author contribution All authors made substantial contributions to the conception or design of the work; drafted the work or revised it critically for important intellectual content; approved the final version; and agreed to be accountable for all aspects of the work in ensuring that questions related to the accuracy or integrity of any part of the work are appropriately investigated and resolved. AF drafted the review concept; FL performed the literature search, wrote the first manuscript, and drafted the tables and figures. AF and GKW made critical comments and revised the manuscript.
}

Funding Open access funding provided by University of Innsbruck and Medical University of Innsbruck. FL is supported by the Stichting ParkinsonFonds and the US Multiple System Atrophy Coalition.

Availability of data and material Data sharing is not applicable to this article as no datasets were generated or analyzed during the current study.

\section{Declarations}

Ethics approval The authors are committed to uphold the integrity of scientific records and adhered to the COPE guidelines. Due to the type of this work, neither ethics approval nor informed consent was required.

Consent to participate Not applicable.

Consent for publication Not applicable.

Conflict of interest FL declares no competing interests. GKW reports honoraria from Biogen, Biohaven, Minoryx, Lundbeck, and Theravance as well as grant support of the Dr. Johannes und Hertha Tuba-Stiftung, outside of the submitted work. AF reports royalties from Springer Verlag, Springer Nature, IOS Press, Impact Medicom, and Thieme Verlag; speaker fees and honoraria from the International Parkinson Disease and Movement Disorder Society, Austrian Autonomic Society, Austrian Parkinson Society, Austrian Neurology Society, Ordensklinikum Linz, Theravance Biopharma, and Abbvie; and research grants from the Stichting ParkinsonFond, the US MSA Coalition, the Dr. Johannes und Hertha Tuba-Stiftung, and the Österreichischer Austauschdienst, outside of the submitted work.

Open Access This article is licensed under a Creative Commons Attribution 4.0 International License, which permits use, sharing, adaptation, distribution and reproduction in any medium or format, as long as you give appropriate credit to the original author(s) and the source, provide a link to the Creative Commons licence, and indicate if changes were made. The images or other third party material in this article are included in the article's Creative Commons licence, unless indicated otherwise in a credit line to the material. If material is not included in the article's Creative Commons licence and your intended use is not permitted by statutory regulation or exceeds the permitted use, you will need to obtain permission directly from the copyright holder. To view a copy of this licence, visit http://creativecommons.org/licenses/by/4.0/.

\section{References}

1. Fanciulli A, Strano S, Colosimo C, Caltagirone C, Spalletta G, Pontieri FE (2013) "The potential prognostic role of cardiovascular autonomic failure in $\alpha$-synucleinopathies," (in eng). Eur J Neurol 20(2):231-235

2. Kaufmann H et al (2017) "Natural history of pure autonomic failure: a United States prospective cohort," (in eng). Ann Neurol 81(2):287-297

3. Papp MI, Kahn JE, Lantos PL (1989) "Glial cytoplasmic inclusions in the CNS of patients with multiple system atrophy (striatonigral degeneration, olivopontocerebellar atrophy and ShyDrager syndrome)," (in eng). J Neurol Sci 94(1-3):79-100

4. Gilman S et al (2008) "Second consensus statement on the diagnosis of multiple system atrophy," (in eng). Neurology 71(9):670-676 
5. Poewe W et al (2017) "Parkinson disease," (in eng). Nat Rev Dis Primers 3:17013

6. Hague K, Lento P, Morgello S, Caro S, Kaufmann H (1997) “The distribution of Lewy bodies in pure autonomic failure: autopsy findings and review of the literature," (in eng). Acta Neuropathol 94(2):192-196

7. Kaufmann H, Hague K, Perl D (2001) "Accumulation of alphasynuclein in autonomic nerves in pure autonomic failure," (in eng). Neurology 56(7):980-981

8. Freeman R et al (2011) "Consensus statement on the definition of orthostatic hypotension, neurally mediated syncope and the postural tachycardia syndrome," (in eng). Clin Auton Res 21(2):69-72

9. R. Freeman, "Clinical practice. Neurogenic orthostatic hypotension," (in eng), N Engl J Med, vol. 358, no. 6, pp. 615-24, 2008.

10. Freeman R, Abuzinadah AR, Gibbons C, Jones P, Miglis MG, Sinn DI (2018) "Orthostatic hypotension: JACC state-of-the-art review," (in eng). J Am Coll Cardiol 72(11):1294-1309

11. Wakabayashi K, Takahashi H (1997) "Neuropathology of autonomic nervous system in Parkinson's disease," (in eng). Eur Neurol 38(Suppl 2):2-7

12. Nakamura K et al (2016) " $\alpha$-Synuclein pathology in the cranial and spinal nerves in Lewy body disease," (in eng). Neuropathology 36(3):262-269

13. Brignole $\mathrm{M}$ et al (2018) $2018 \mathrm{ESC}$ Guidelines for the diagnosis and management of syncope. Eur Heart J 39(21):1883-1948

14. Norcliffe-Kaufmann L et al (2018) "Orthostatic heart rate changes in patients with autonomic failure caused by neurodegenerative synucleinopathies," (in eng). Ann Neurol 83(3):522-531

15. Velseboer DC, de Haan RJ, Wieling W, Goldstein DS, de Bie RM (2011) "Prevalence of orthostatic hypotension in Parkinson's disease: a systematic review and meta-analysis," (in eng). Parkinsonism Relat Disord 17(10):724-729

16. Lees AJ, Hardy J, Revesz T (2009) "Parkinson's disease," (in eng). Lancet 373(9680):2055-2066

17. Wenning GK et al (2013) "The natural history of multiple system atrophy: a prospective European cohort study," (in eng). Lancet Neurol 12(3):264-274

18. Low PA et al (2015) Natural history of multiple system atrophy in the USA: a prospective cohort study. Lancet Neurol 14(7):710-719

19. McKeith IG et al (2017) "Diagnosis and management of dementia with Lewy bodies: Fourth consensus report of the DLB Consortium," (in eng). Neurology 89(1):88-100

20. Thaisetthawatkul P et al (2004) "Autonomic dysfunction in dementia with Lewy bodies," (in eng). Neurology 62(10):1804-1809

21. K. Stubendorff, D. Aarsland, L. Minthon, and E. Londos, "The impact of autonomic dysfunction on survival in patients with dementia with Lewy bodies and Parkinson's disease with dementia," (in eng), PLoS One, vol. 7, no. 10, p. e45451, 2012.

22. "Consensus statement on the definition of orthostatic hypotension, pure autonomic failure, and multiple system atrophy. The Consensus Committee of the American Autonomic Society and the American Academy of Neurology," (in eng), Neurology, vol. 46, no. 5, p. 1470, May 1996.

23. A. Fanciulli et al., "Consensus statement on the definition of neurogenic supine hypertension in cardiovascular autonomic failure by the American Autonomic Society (AAS) and the European Federation of Autonomic Societies (EFAS) : Endorsed by the European Academy of Neurology (EAN) and the European Society of Hypertension (ESH)," (in eng), Clin Auton Res, May 2018.

24. Jansen RW, Lipsitz LA (1995) "Postprandial hypotension: epidemiology, pathophysiology, and clinical management," (in eng). Ann Intern Med 122(4):286-295
25. Low DA, da Nóbrega AC, Mathias CJ (2012) "Exercise-induced hypotension in autonomic disorders," (in eng). Auton Neurosci 171(1-2):66-78

26. Fanciulli A, Wenning GK (2015) "Multiple-system atrophy," (in eng). N Engl J Med 372(14):1375-6

27. Palma JA, Norcliffe-Kaufmann L, Kaufmann H (2018) "Diagnosis of multiple system atrophy," (in eng). Auton Neurosci 211:15-25

28. Garland EM, Hooper WB, Robertson D (2013) "Pure autonomic failure," (in eng). Handb Clin Neurol 117:243-257

29. Singer W et al (2017) "Pure autonomic failure: Predictors of conversion to clinical CNS involvement," (in eng). Neurology 88(12):1129-1136

30. Coon EA et al (2020) "Predicting phenoconversion in pure autonomic failure," (in eng). Neurology 95(7):e889-e897

31. Giannini G et al (2018) "The natural history of idiopathic autonomic failure: The IAF-BO cohort study," (in eng). Neurology 91(13):e1245-e1254

32. Baschieri F et al (2017) "Iodine-123-meta-iodobenzylguanidine Myocardial Scintigraphy in Isolated Autonomic Failure: Potential Red Flag for Future Multiple System Atrophy," (in eng). Front Neurol 8:225

33. Singer W et al (2021) "Alpha-synuclein oligomers and neurofilament light chain predict phenoconversion of pure autonomic failure," (in eng). Ann Neurol 89(6):1212-1220

34. Pellecchia MT et al (2020) "Can autonomic testing and imaging contribute to the early diagnosis of multiple system atrophy? A Systematic Review and Recommendations by the Movement Disorder Society Multiple System Atrophy Study Group," (in eng). Mov Disord Clin Pract 7(7):750-762

35. Fanciulli A et al (2017) "Very late-onset pure autonomic failure," (in eng). Mov Disord 32(7):1106-1108

36. Singer W et al (2020) "Alpha-synuclein oligomers and neurofilament light chain in spinal fluid differentiate multiple system atrophy from Lewy body synucleinopathies," (in eng). Ann Neurol 88(3):503-512

37. Keener AM, Bordelon YM (2016) "Parkinsonism," (in eng). Semin Neurol 36(4):330-334

38. Levin J, Kurz A, Arzberger T, Giese A, Höglinger GU (2016) "The differential diagnosis and treatment of atypical parkinsonism," (in eng). Deutsches Arzteblatt international 113(5):61-69

39. Höglinger GU et al (2017) "Clinical diagnosis of progressive supranuclear palsy: the movement disorder society criteria," (in eng). Mov Disord 32(6):853-864

40. Armstrong MJ et al (2013) "Criteria for the diagnosis of corticobasal degeneration," (in eng). Neurology 80(5):496-503

41. Miki Y et al (2019) "Improving diagnostic accuracy of multiple system atrophy: a clinicopathological study," (in eng). Brain 142(9):2813-2827

42. Schmidt $\mathrm{C}$ et al (2008) "Autonomic dysfunction in patients with progressive supranuclear palsy," (in eng). Mov Disord 23(14):2083-2089

43. Holmberg B, Kallio M, Johnels B, Elam M (2001) "Cardiovascular reflex testing contributes to clinical evaluation and differential diagnosis of Parkinsonian syndromes," (in eng). Mov Disord 16(2):217-225

44. Schmidt C et al (2009) "Loss of nocturnal blood pressure fall in various extrapyramidal syndromes," (in eng). Mov Disord 24(14):2136-2142

45. Kimber J et al (2000) "Physiological, pharmacological and neurohormonal assessment of autonomic function in progressive supranuclear palsy," (in eng). Brain 123(Pt 7):1422-1430

46. Wenning GK et al (1999) "Time course of symptomatic orthostatic hypotension and urinary incontinence in patients with postmortem confirmed parkinsonian syndromes: a 
clinicopathological study," (in eng). J Neurol Neurosurg Psychiatry $67(5): 620-623$

47. van Gerpen JA et al (2019) "Progressive supranuclear palsy is not associated with neurogenic orthostatic hypotension," (in eng). Neurology 93(14):e1339-e1347

48. Palermo G, Del Prete E, Bonuccelli U, Ceravolo R (2020) "Early autonomic and cognitive dysfunction in PD, DLB and MSA: blurring the boundaries between $\alpha$-synucleinopathies," (in eng). J Neurol 267(12):3444-3456

49. Koga $\mathrm{S}$ et al (2015) "When DLB, PD, and PSP masquerade as MSA: an autopsy study of 134 patients," (in eng). Neurology 85(5):404-412

50. Fanciulli A et al (2014) "Detecting nocturnal hypertension in Parkinson's disease and multiple system atrophy: proposal of a decision-support algorithm," (in eng). J Neurol 261(7):1291-1299

51. F. Leys, A. Fanciulli, J. P. Ndayisaba, R. Granata, W. Struhal, and G. K. Wenning, "Cardiovascular autonomic function testing in multiple system atrophy and Parkinson's disease: an expert-based blinded evaluation," (in eng), Clin Auton Res, 2020.

52. Fanciulli A et al (2019) "Early distinction of Parkinson-variant multiple system atrophy from Parkinson's disease," (in eng). Mov Disord 34(3):440-441

53. Fanciulli A et al (2019) "Urinary retention discriminates multiple system atrophy from Parkinson's disease,” (in eng). Mov Disord 34(12):1926-1928

54. Lenka A, Lamotte G, Goldstein DS (2021) "Cardiac (18) F-Dopamine PET distinguishes PD with orthostatic hypotension from Parkinsonian MSA," (in eng). Mov Disord Clin Pract 8(4):582-586

55. Orimo S, Yogo M, Nakamura T, Suzuki M, Watanabe H (2016) "(123)I-meta-iodobenzylguanidine (MIBG) cardiac scintigraphy in $\alpha$-synucleinopathies," (in eng). Ageing Res Rev 30:122-133

56. Orimo S, Suzuki M, Inaba A, Mizusawa H (2012) "123I-MIBG myocardial scintigraphy for differentiating Parkinson's disease from other neurodegenerative parkinsonism: a systematic review and meta-analysis," (in eng). Parkinsonism Relat Disord 18(5):494-500

57. Goldstein DS, Cheshire WP (2018) Roles of cardiac sympathetic neuroimaging in autonomic medicine. Clin Auton Res 28(4):397-410

58. Goldstein DS (2014) "Dysautonomia in Parkinson disease," (in eng). Compr Physiol 4(2):805-826

59. Postuma RB et al (2015) "MDS clinical diagnostic criteria for Parkinson's disease," (in eng). Mov Disord 30(12):1591-1601

60. Fanciulli A et al (2016) "Supine hypertension in Parkinson's disease and multiple system atrophy," (in eng). Clin Auton Res 26(2):97-105

61. Gale SA, Acar D, Daffner KR (2018) “Dementia," (in eng). Am J Med 131(10):1161-1169

62. E. C. Finger, "Frontotemporal Dementias," (in eng), Continuum (Minneapolis, Minn.), vol. 22, no. 2 Dementia, pp. 464-89, Apr 2016.

63. Allan LM et al (2007) "Autonomic dysfunction in dementia," (in eng). J Neurol Neurosurg Psychiatry 78(7):671-677

64. Struhal W et al (2014) "The phoenix from the ashes: cardiovascular autonomic dysfunction in behavioral variant of frontotemporal dementia," (in eng). J Alzheimer's Dis 42(3):1041-1046

65. Struhal W et al (2016) "Heart rate spectra confirm the presence of autonomic dysfunction in dementia patients," (in eng). Journal of Alzheimer's disease : JAD 54(2):657-667

66. Jensen-Dahm C et al (2015) "Autonomic dysfunction in patients with mild to moderate Alzheimer's disease," (in eng). Journal of Alzheimer's disease : JAD 47(3):681-689

67. Idiaquez J, Roman GC (2011) "Autonomic dysfunction in neurodegenerative dementias," (in eng). J Neurol Sci 305(1-2):22-27
68. Allan LM (2019) Diagnosis and management of autonomic dysfunction in dementia syndromes. Curr Treat Options Neurol 21(8):38

69. Idiaquez J, Rios L, Sandoval E (1997) "Postprandial hypotension in Alzheimer's disease," (in eng). Clin Auton Res 7(3):119-120

70. D. Tulbă, L. Cozma, B. O. Popescu, and E. I. Davidescu, "Dysautonomia in Alzheimer's disease," (in eng), Medicina (Kaunas, Lithuania), vol. 56, no. 7, 2020.

71. Toru S, Kanouchi T, Yokota T, Yagi Y, Machida A, Kobayashi $\mathrm{T}$ (2018) "Utility of autonomic function tests to differentiate dementia with Lewy bodies and parkinson disease with dementia from Alzheimer disease," (in eng). Eur Neurol 79(1-2):27-32

72. Stankovic I et al (2014) "Cognitive impairment in multiple system atrophy: a position statement by the Neuropsychology Task Force of the MDS Multiple System Atrophy (MODIMSA) study group," (in eng). Mov Disord 29(7):857-867

73. Akbar U, Ashizawa T (2015) "Ataxia," (in eng). Neurol Clin 33(1):225-248

74. Klockgether T (2010) "Sporadic ataxia with adult onset: classification and diagnostic criteria," (in eng). Lancet Neurol 9(1):94-104

75. Lin DJ, Hermann KL, Schmahmann JD (2016) "The diagnosis and natural history of multiple system atrophy, cerebellar type," (in eng). Cerebellum (London, England) 15(6):663-679

76. Indelicato E et al (2020) "Cardiovascular autonomic testing in the work-up of cerebellar ataxia: insight from an observational single center study," (in eng). J Neurol 267(4):1097-1102

77. Gilman S et al (2000) "Evolution of sporadic olivopontocerebellar atrophy into multiple system atrophy," (in eng). Neurology 55(4):527-532

78. Abele M, Minnerop M, Urbach H, Specht K, Klockgether T (2007) "Sporadic adult onset ataxia of unknown etiology : a clinical, electrophysiological and imaging study," (in eng). J Neurol 254(10):1384-1389

79. Leehey MA (2009) "Fragile X-associated tremor/ataxia syndrome: clinical phenotype, diagnosis, and treatment," (in eng), Journal of investigative medicine : the official publication of the American Federation for. Clin Res 57(8):830-836

80. Kamm C et al (2005) "The fragile $X$ tremor ataxia syndrome in the differential diagnosis of multiple system atrophy: data from the EMSA Study Group," (in eng). Brain 128(Pt 8):1855-1860

81. Hagerman R, Hagerman P (2013) "Advances in clinical and molecular understanding of the FMR1 premutation and fragile $\mathrm{X}$-associated tremor/ataxia syndrome," (in eng). Lancet Neurol 12(8):786-798

82. Indelicato E et al (2018) "Autonomic function testing in Friedreich's ataxia," (in eng). J Neurol 265(9):2015-2022

83. Ingall TJ, McLeod JG (1991) "Autonomic function in Friedreich's ataxia," (in eng). J Neurol Neurosurg Psychiatry 54(2):162-164

84. A. Cortese, M. M. Reilly, and H. Houlden, "RFC1 CANVAS / Spectrum Disorder," in GeneReviews( $\left({ }^{\circ}\right)$, M. P. Adam et al., Eds. Seattle (WA): University of Washington, Seattle. Copyright (C) 1993-2021, University of Washington, Seattle. GeneReviews is a registered trademark of the University of Washington, Seattle. All rights reserved., 2020.

85. Cortese A et al (2019) "Biallelic expansion of an intronic repeat in RFC1 is a common cause of late-onset ataxia," (in eng). Nat Genet 51(4):649-658

86. Cortese A et al (2020) "Cerebellar ataxia, neuropathy, vestibular areflexia syndrome due to RFC1 repeat expansion," (in eng). Brain 143(2):480-490

87. Fan Y et al (2020) "No biallelic intronic AAGGG repeat expansion in RFC1 was found in patients with late-onset ataxia and MSA," (in eng). Parkinsonism Relat Disord 73:1-2 
88. Kim HJ, Stamelou M, Jeon B (2016) "Multiple system atrophymimicking conditions: diagnostic challenges," (in eng). Parkinsonism Relat Disord 22(Suppl 1):S12-S15

89. Kim HJ et al (2014) "Should genetic testing for SCAs be included in the diagnostic workup for MSA?," (in eng). Neurology 83(19): 1733-1738

90. Indelicato E et al (2018) "Autonomic function testing in spinocerebellar ataxia type 2," (in eng). Clin Auton Res 28(3):341-346

91. Takazaki KA, D’Abreu A, Nucci A, Lopes-Cendes I, França MC $\mathrm{Jr}$ (2013) "Dysautonomia is frequent in Machado-Joseph disease: clinical and neurophysiological evaluation," (in eng). Cerebellum (London, England) 12(4):513-519

92. Kazuta T, Hayashi M, Shimizu T, Iwasaki A, Nakamura S, Hirai S (2000) "Autonomic dysfunction in Machado-Joseph disease assessed by iodine123-labeled metaiodobenzylguanidine myocardial scintigraphy," (in eng). Clin Auton Res 10(3):111-115

93. Fanciulli A, Leys F, Falup-Pecurariu C, Thijs R, Wenning GK (2020) "Management of orthostatic hypotension in Parkinson's disease," (in eng). J Parkinsons Dis 10(s1):S57-s64

94. Freeman R (2006) "Assessment of cardiovascular autonomic function," (in eng). Clin Neurophysiol 117(4):716-730

95. Hilz MJ, Dütsch M (2006) "Quantitative studies of autonomic function," (in eng). Muscle Nerve 33(1):6-20

96. Cygankiewicz I, Zareba W (2013) "Heart rate variability," (in eng). Handb Clin Neurol 117:379-393

97. Cheshire WP et al (2021) "Electrodiagnostic assessment of the autonomic nervous system: a consensus statement endorsed by the American Autonomic Society, American Academy of Neurology, and the International Federation of Clinical Neurophysiology," (in eng). Clin Neurophysiol 132(2):666-682

98. Thijs RD et al (2021) Recommendations for tilt table testing and other provocative cardiovascular autonomic tests in conditions that may cause transient loss of consciousness: consensus statement of the European Federation of Autonomic Societies (EFAS) endorsed by the American Autonomic Society (AAS) and the European Academy of Neurology (EAN)," (in eng). Auton Neurosci 233:102792

99. J. A. Palma and H. Kaufmann, "Management of Orthostatic Hypotension," (in eng), Continuum (Minneapolis, Minn.), vol. 26, no. 1, pp. 154-177, 2020.

100. Gupta V, Lipsitz LA (2007) "Orthostatic hypotension in the elderly: diagnosis and treatment," (in eng). Am J Med 120(10):841-847

101. Fanciulli A, Campese N, Wenning GK (2019) "The Schellong test: detecting orthostatic blood pressure and heart rate changes in German-speaking countries," (in eng). Clin Auton Res 29(4):363-366

102. A. Fanciulli et al., "Validation of the neurogenic orthostatic hypotension ratio with active standing," (in eng), Ann Neurol, 2020.

Publisher's Note Springer Nature remains neutral with regard to jurisdictional claims in published maps and institutional affiliations. 